\title{
Uma revisão sistemática sobre os benefícios da drenagem linfática manual no tratamento do edema em membros inferiores
}

\author{
A systematic review about benefits of manual lymphatic drainage in treating edema in lower \\ members
}

Una revisión sistemática sobre beneficios del drenaje linfático manual en el tratamiento del edema en miembros inferiores

Recebido: 16/03/2021 | Revisado: 23/03/2021 | Aceito: 26/03/2021 | Publicado: 03/04/2021

\author{
Paloma Kassia Sousa de Brito \\ ORCID: https://orcid.org/0000-0002-1033-9687 \\ Centro Universitário FIBRA, Brasil \\ E-mail: palomaa.kassia@gmail.com \\ Carolina Cabral Angelim \\ ORCID: https://orcid.org/0000-0003-2195-0366 \\ Centro Universitário FIBRA, Brasil \\ E-mail: carolina.995121@gmail.com \\ Samir Mansour Moraes Casseb \\ ORCID: https://orcid.org/0000-0002-7419-3381 \\ Centro Universitário FIBRA, Brasil \\ E-mail: samir.casseb@gmail.com
}

\begin{abstract}
Resumo
A drenagem linfática manual (DLM), consiste em uma técnica de manobras utilizadas em tratamentos fisioterápicos, a fim de direcionar e aumentar o fluxo linfático suavemente. O procedimento é considerado uma alternativa para o tratamento de edemas em membros inferiores causados, em sua maioria, pelo envelhecimento e insuficiências (cardíaca, hepática, linfática, renal e venosa). Nesse sentido, o objetivo é apresentar os benefícios do tratamento de edema em membros inferiores através da DLM. Para este fim, foram utilizados dados bibliográficos extraídos de artigos em português e inglês publicados entre os anos de 1995-2019 nas bases de dados: LILACS, Scielo, MEDLINE e BVS. Utilizando as palavras "edema", "drenagem linfática" e "membros inferiores", foram obtidos 52 artigos. Dentre eles, 8 se encaixavam nos critérios deste estudo e foram selecionados para análise. Os trabalhos apresentaram as condições dos pacientes antes e após a terapia. Verificou-se nos artigos que todos os pacientes possuíam comprometimento circulatório ou linfático que geravam edemas. Em decorrência disso, passaram por estímulo e evacuação de linfonodos, seguindo movimentos com pressão rítmica, lenta e suave, além de medidas de compressão da região afetada. Essa terapia mostrou-se eficiente no tratamento de dermatofibrose, flebite, doença venosa crônica, fibroedema geloide e linfedemas; promovendo redução do edema e alívio das dores.
\end{abstract}

Palavras-chave: Edema; Terapia; Patologia.

\begin{abstract}
Manual lymphatic drainage (DLM), consists of a maneuver technique used in physiotherapy treatments, in order to smoothly direct and increase lymphatic flow. The procedure is considered an alternative for the treatment of edema in the lower limbs, mostly caused by aging and insufficiencies (cardiac, hepatic, lymphatic, renal and venous). In this sense, the objective is to present the benefits of treating lower limb edema through MLD. For this purpose, bibliographic data extracted from articles in Portuguese and English published between 1995-2019 in the databases: LILACS, Scielo, MEDLINE and BVS were used. Using the words "edema", "lymphatic drainage" and "lower limbs", 52 articles were obtained. Among them, 8 fit the criteria of this study and were analyzed. The studies presented the conditions of patients before and after therapy. It was found in the articles that all patients had circulatory or lymphatic involvement that generated edema. As a result, they underwent stimulation and evacuation of lymph nodes, following movements with rhythmic pressure, slow and smooth, in addition to measures of compression on the affected region. This therapy proved to be efficient in the treatment of dermatofibrosis, phlebitis, chronic venous disease, iceloid fibroedema and lymphedemas; promoting edema reduction and pain relief.
\end{abstract}

Keywords: Edema; Therapy; Pathology. 


\section{Resumen}

El drenaje linfático manual (DLM), consiste en una técnica de maniobra utilizada en los tratamientos de fisioterapia, con el fin de dirigir y aumentar suavemente el flujo linfático. El procedimiento se considera una alternativa para el tratamiento del edema en los miembros inferiores, mayoritariamente causado por el envejecimiento y las insuficiencias (cardíacas, hepáticas, linfáticas, renales y venosas). En este sentido, el objetivo es presentar los beneficios del tratamiento del edema de miembros inferiores mediante DLM. Para ello se utilizaron datos bibliográficos extraídos de artículos en portugués e inglés publicados entre 1995-2019 en las bases de datos: LILACS, Scielo, MEDLINE y VHL. Utilizando las palabras “edema", "drenaje linfático" y "miembros inferiores" se obtuvieron 52 artículos. Entre ellos, 8 cumplieron los criterios de este estudio y fueron analizados. Los estudios presentaron las condiciones de los pacientes antes y después de la terapia. En los artículos se encontró que todos los pacientes presentaban afectación circulatoria o linfática que generaba edema. Como resultado, se sometieron a estimulación y evacuación de los ganglios linfáticos, siguiendo movimientos con presión rítmica, lenta y suave, además de medidas de compresión de la región afectada. Esta terapia demostró ser eficaz en el tratamiento de la dermatofibrosis, flebitis, enfermedad venosa crónica, fibroedema icloide y linfedemas; promover la reducción del edema y el alivio del dolor.

Palabras clave: Edema; Terapia; Patología.

\section{Introdução}

A drenagem linfática manual (DLM), consiste em uma técnica de manobras utilizadas em tratamentos fisioterápicos, a fim de direcionar e aumentar o fluxo linfático suavemente (Soares, 2012). Portanto, este recurso terapêutico é fundamental para estimular a circulação sanguínea, eliminar toxinas e nutrir os tecidos, conduzindo o líquido intersticial até os gânglios linfáticos para que sejam eliminados pela urina (Franca et al., 2013). Este procedimento é bastante conhecido atualmente, é muito utilizado no tratamento de edema. Nesse sentido, torna-se fundamental a compreensão das situações que podem favorecer esse extravasamento de líquido dos vasos para os tecidos. É importante destacar que os pacientes geralmente apresentam mais de um mecanismo de complicações ao mesmo tempo, bem como: inflamação dos vasos ou tecidos; aumento da pressão dentro das veias e capilares; redução da pressão oncótica; linfedema (Pinheiro, 2020).

Segundo Lopes, 2006, esta técnica foi desenvolvida pelo biólogo e fisioterapeuta Dr. Phil Emil Vodderum na década de 30. A partir de então, outros pesquisadores desenvolveram a base científica deste procedimento, como Földi e Kehnke (Alemanha), Cashley-Smith (Austrália) e Leduc (Bélgica) (Zanella et al, 2011). Porém, houveram algumas diferenças em relação ao tipo de movimento, enquanto Vooder trabalhava com movimentos circulares, rotatórios e de bombeio, Leduc utilizava um método mais restrito (Piccinini et al., 2009). As duas técnicas foram classificadas como eficazes para serem utilizadas como terapia, pois abordam as manobras mais essenciais da DLM: captação, reabsorção e evacuação da linfa; realizadas com leves pressões em movimentos circulares (Guirro \& Guirro, 2004).

O edema é uma disfunção que consiste no acúmulo anormal de líquido no espaço extracelular intersticial ou nas cavidades corporais devido ao mau funcionamento do sistema linfático, composto por uma solução aquosa de sais e proteínas do plasma, e sua composição depende da causa que levou à formação desta patologia. Quando o líquido se acumula no corpo inteiro, diz-se que é um edema generalizado (Ozolins, 2018). Vários mecanismos são responsáveis por ocasionar sua formação, bem como: a diminuição da pressão oncótica; o aumento da pressão hidrostática; o aumento da permeabilidade capilar; o aumento da pressão venosa; e o depósito tecidual. O edema em membros inferiores é bastante comum e pode ser de sério risco à saúde, podendo se manifestar de forma unilateral ou bilateral (Goroll \& Mulley, 2006). Devido a isso, é essencial que seja realizado uma anamnese e exame físico para comprovação da existência do edema e, para isso, existem mecanismos que podem auxiliar no diagnóstico desta enfermidade, para que não seja confundido com linfedema.

É fundamental informar ao paciente que nem sempre apenas diuréticos devem ser prescritos, pois eles apenas servem se à causa do edema por retenção de líquidos, caso o paciente manifeste uma trombose, edema linfático, infecção ou qualquer outro fator que não seja o acúmulo de líquidos, os diuréticos podem ter efeitos mais negativos do que positivos, por esse motivo é essencial entender o que ocasionou a formação do inchaço antes de indicar o tratamento (Pinheiro, 2020). O primeiro passo é confirmar a presença do edema através da formação do sinal de Godet, é necessário pressionar com o polegar a região pré-tibial 
e maleolar os membros inferiores por, pelo menos, cinco segundos; em seguida, verifica-se o sinal de Stemmer, que pode ser encontrado ao pressionar a pele na base do segundo artelho do membro acometido (Trayes et al., 2013). A partir desses procedimentos, se o examinador não conseguir fazer a preensão na região suspeita de edema, é possível que o sinal seja considerado positivo (Filho et al., 2013).

A DLM é importante para o alívio de dores, circulação sanguínea, edemas, hipertensão arterial, tecido edemaciado, reumatismo, tensão menstrual, musculatura tensa, pele irritada, sistema nervoso abalado. A aplicação deste recurso deve ser realizada de maneira lenta, rítmica e suave, de modo que não cause danos ou lesões teciduais ao paciente, em razão disso, é necessário efetuar o procedimento conforme a anatomia e fisiologia do sistema linfático (Tacani \& Tacani, 2008). Na Figura 1 é possível identificar como ocorre o procedimento de drenagem nos membros inferiores do paciente, para o tratamento de edema.

Figura 1. Procedimento de drenagem linfática manual nos membros inferiores.

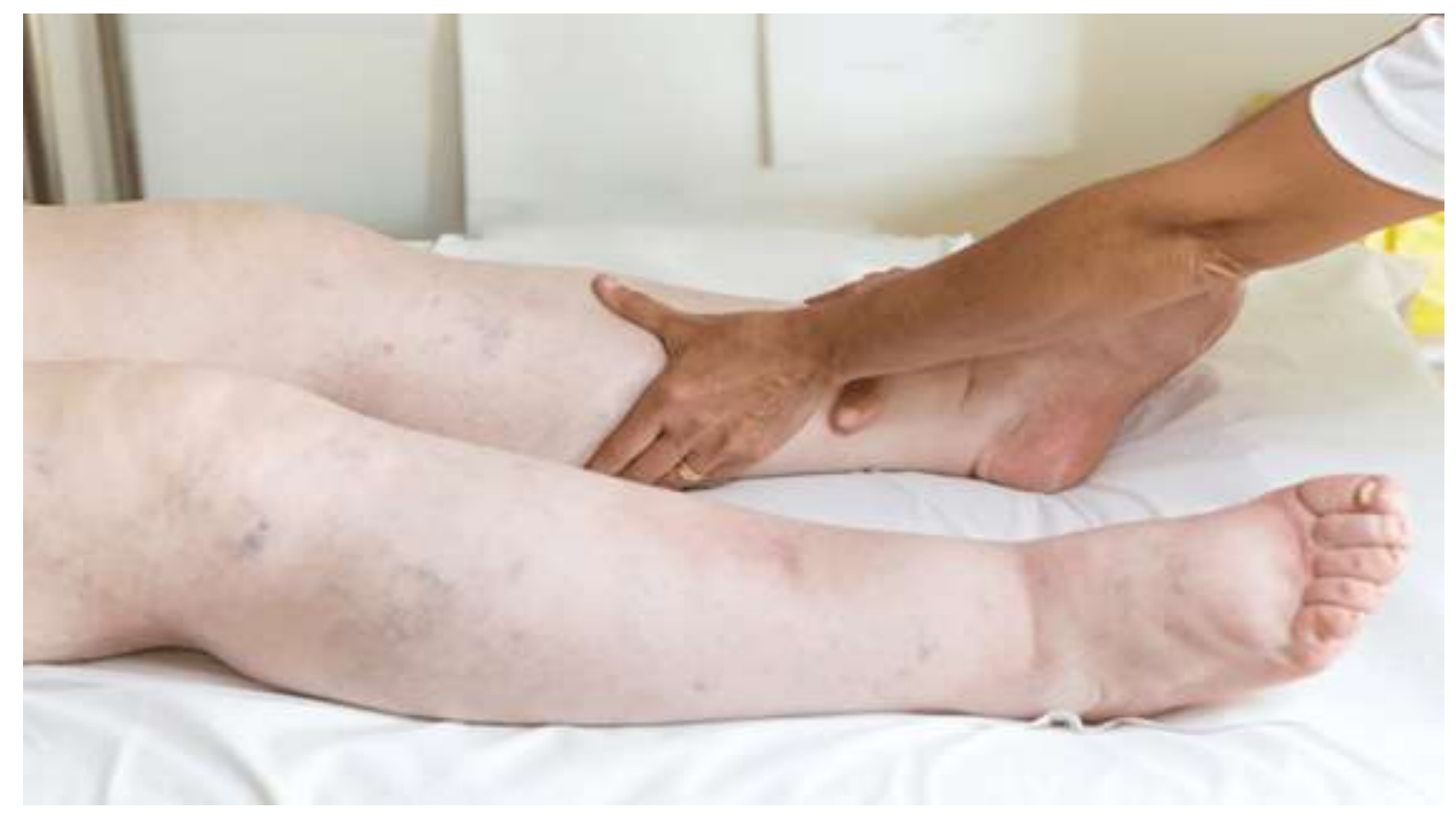

Fonte: EducarSaude (2009).

A imagem demonstra a técnica de DLM em membros inferiores do paciente, para o tratamento de edema. É perceptível que as regiões tratadas são internas, visando atingir os canais linfáticos e linfonodos, para se adequar às necessidades do paciente. A partir destas considerações teóricas e conceituais, os objetivos do presente estudo é apresentar os benefícios do tratamento de edema em membros inferiores através da DLM a partir da análise de estudos sobre essa temática.

\section{Metodologia}

Foram pesquisados artigos publicados no período entre 1995 a 2019. As bases de dados utilizadas para esta pesquisa foram Scielo, Lilacs, Medline, além de livros e periódicos. Foram utilizados os termos: drenagem linfática; edema; membros inferiores. Como critérios de inclusão, os trabalhos deveriam apresentar a técnica DLM no tratamento de edemas decorrentes de patologias em membros inferiores (insuficiências, linfedemas, fibroedemas, etc). Como critérios de exclusão, descartamos os trabalhos que apresentassem o uso da DLM para tratamento de pacientes com tumores benignos ou malignos em membros inferiores; de igual modo, excluímos aqueles que abordavam uso de DLM na região facial em procedimentos ortodônticos, haja vista que não davam enfoque à região de interesse deste estudo. 
Este trabalho consiste em uma revisão sistemática de caráter quantitativo segundo os parâmetros de De-la-Torre-UgarteGuanilo et al (2011), haja vista que busca avaliar a eficácia da DLM enquanto terapia, destacando os benefícios de sua utilização. Além disso, utilizamos três trabalhos no mesmo modelo (revisão sistemática) para embasar a organização de nossa metodologia e resultados. Foram eles: Efeitos do exercício físico sobre diabetes mellitus tipo 1: uma revisão sistemática de ensaios clínicos e randomizados (Marçal et al., 2018); Intervenções escolares para redução da obesidade infantil: uma revisão sistemática (Andrade et al., 2015); Peso ao nascer e obesidade em crianças e adolescentes: uma revisão sistemática (Rossi \& Vasconcelos, 2010).

\section{Resultados e Discussão}

A busca retornou em 52 artigos, dentre os quais 24 artigos foram eliminados segundo os critérios de exclusão, e 11 foram aceitos pelos critérios de inclusão. Após a leitura e análise de resumos dos artigos aceitos, 8 foram incluídos neste estudo (Figura 2)

Figura 2. Fluxograma da seleção de trabalhos.

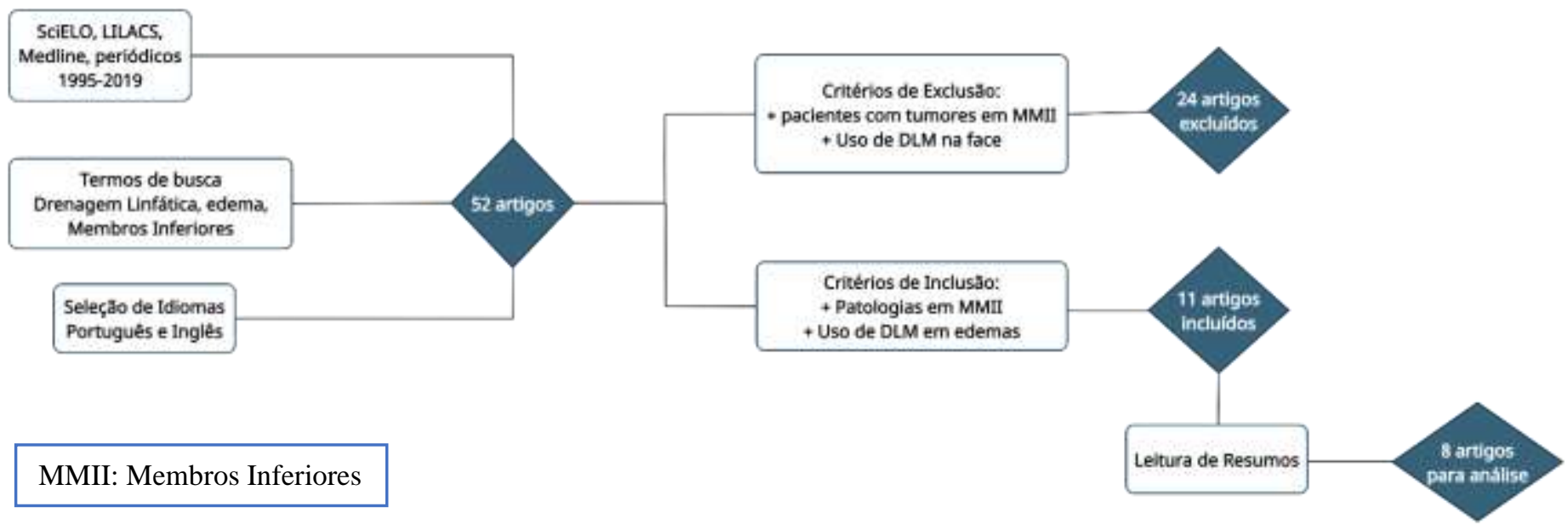

Fonte: Autores.

Dentre os artigos selecionados para embasar este estudo estão presentes 2 relatos de caso; 1 revisão retrospectiva; 2 ensaios clínicos; 2 estudos observacionais, sendo um de coorte e o outro série de Casos; e um estudo classificado como quaseexperimental. O resumo destes trabalhos se encontra na Tabela 1. 
Tabela 1. Resumo dos trabalhos.

\begin{tabular}{|c|c|c|c|c|}
\hline Referência & Tipo/tempo & Sintomatologia & Intervenção & Resultados \\
\hline $\begin{array}{l}\text { Valente, Godoy } \\
\text { e Godoy (2009) }\end{array}$ & $\begin{array}{l}\text { Relato de caso, } \\
\text { mulher de } 73 \text { anos }\end{array}$ & $\begin{array}{l}\text { Insuficiência Venosa Crônica } \\
\text { (IVC) e Dermatofibrose } \\
\text { associada à Flebite }\end{array}$ & $\begin{array}{l}\text { DL.M Godoy \& Godoy e drenagem } \\
\text { linfática meclinica (RA/3000 Godoy) } \\
\text { durante } 5 \text { dias, não consecutivos, com } \\
\text { duraclio de / lh cada sessllo. }\end{array}$ & $\begin{array}{l}\text { clareamento progressivo da pele, } \\
\text { reduçalo da sensibilidade dolorosa local } \\
\text { e redução do edema }\end{array}$ \\
\hline $\begin{array}{l}\text { Soligo, et al. } \\
\qquad(2008)\end{array}$ & $\begin{array}{l}\text { Relato de caso, } \\
\text { mulher de } 33 \text { anos }\end{array}$ & $\begin{array}{l}\text { Linfedema Traumático em } \\
\text { embro inferior direito }\end{array}$ & $\begin{array}{c}\text { DL.M Godoy \& Godoy', } 5 \text { vezes por } \\
\text { semana, ith por dia }\end{array}$ & $\begin{array}{l}\text { reduçâo clinica do linfedema } \\
\text { confirmado pela perimetria e volumetria }\end{array}$ \\
\hline $\begin{array}{l}\text { Kostanog̣lu, } \\
\text { Ramoḡlu e } \\
\text { Güneren (2019 }\end{array}$ & $\begin{array}{l}\text { Retrospectivo, } 95 \\
\text { pacientes com LEL } \\
\text { entre } 2015 \text { e } 2017\end{array}$ & $\begin{array}{l}\text { Linfedema de Membros } \\
\text { Inferiores }\end{array}$ & $\begin{array}{l}4 \text { semanas com } \mathrm{TDC}^{2} \text { modificada. } \\
\text { incluindo drenagem linfática auto- } \\
\text { manual, auto-bandagem, exercicios } \\
\text { desconestivos }\end{array}$ & $\begin{array}{l}\text { Redução significativa no excesso de } \\
\text { volume da extremidade (EEV) }\end{array}$ \\
\hline $\begin{array}{l}\text { Leal, et al. } \\
\text { (2015) }\end{array}$ & $\begin{array}{l}\text { Estudo de coorte } \\
\text { prospectivo } \\
\text { longitudinal, } 10 \\
\text { pacientes } \\
\end{array}$ & Doença Venosa Crônica & $\begin{array}{l}\text { tratamento fisioterapêtutico vascular e } \\
\text { DLM, em } 10 \text { sessōes de } 1 \mathrm{~h}\end{array}$ & $\begin{array}{c}\text { Eliminaçào do edema, dor local e } \\
\text { prurido }\end{array}$ \\
\hline $\begin{array}{l}\text { Crisótomo, et al. } \\
\qquad(2014)\end{array}$ & $\begin{array}{l}\text { Ensaio clínico } \\
\text { randomizado } \\
\text { simples-cego }\end{array}$ & $\begin{array}{c}\text { Insuficiência Venosa Crônica } \\
\text { (IVC) }\end{array}$ & $\begin{array}{c}10 \text { sessoles de DL.M em MMIP durante } 4 \\
\text { semanas e } 1 \text { sessâo educacional }\end{array}$ & $\begin{array}{c}\text { Melhora a gravidade da IVC e o edema, } \\
\text { os sintomas e a QVRS relacionados à } \\
\text { dor em pacientes }\end{array}$ \\
\hline $\begin{array}{l}\text { Azoubel, et al. } \\
\text { (2010) }\end{array}$ & $\begin{array}{l}\text { Quase experimental, } \\
20 \text { pacientes, de } \\
2007 \text { a } 2008\end{array}$ & Úlceras Venosas & $\begin{array}{l}\text { elevação de MMII a } 30^{\circ} \text {, DL.M, } \\
\text { compressão com bandagens elásticas e } \\
\text { exercícios miolinfocinéticos } 3 \text { vezes com } \\
30 \text { repeticōes }\end{array}$ & $\begin{array}{l}\text { Aceleração do processo cicatricial, } \\
\text { reduçảo da dor e do edema dos } \\
\text { membros afetados. }\end{array}$ \\
\hline $\begin{array}{l}\text { Meyer, et al. } \\
\qquad(2008)\end{array}$ & $\begin{array}{l}\text { Ensaio Clínico } \\
\text { radomizado, } 3 \\
\text { pacientes }\end{array}$ & Fibroedema Geloide - Celulite & $\begin{array}{l}\text { Drenagem linfática - } 20 \text { sessöes, } 3 \text { venes } \\
\text { por semana de DL.M na regiāo anterior e } \\
\text { posterior da coxa e pema. }\end{array}$ & $\begin{array}{l}\text { retirada do excesso de liquido presentes } \\
\text { nos espacos intersticiais melhorando o } \\
\text { processo fibrótico e o contomo da pele }\end{array}$ \\
\hline $\begin{array}{l}\text { Delgado, et al. } \\
\quad(2017)\end{array}$ & $\begin{array}{c}\text { Série de caso, } 10 \\
\text { pacientes no } 2^{\circ} \text { e } 3^{\circ} \\
\text { trimestre }\end{array}$ & Edema gestacional & $\begin{array}{c}\text { sessôes de DL.M, } 2 \text { veres por semana, ith } \\
\text { por dia até a hora do parto }\end{array}$ & $\begin{array}{c}\text { Reduçẩo do edema em até } 5 \mathrm{~cm} \text {, da dor e } \\
\text { da fadiga nos MMII }\end{array}$ \\
\hline
\end{tabular}

${ }^{1}$ Drenagem Linfática Manual Gody\&Godoy: técnica linear, seguindo o trajeto dos vasos linfáticos.

2 Terapia Combinada de Descongestionamento.

${ }^{3}$ Membros Inferiores.

Fonte: Autores.

A insuficiência venosa crônica (IVC) é definida como um distúrbio no desempenho das válvulas do sistema venoso, caracterizando uma incompetência que pode ocasionar obstrução do fluxo venoso (Pena \& Macedo, 2011). Estudos indicam que pelo menos $20 \%$ de homens e mulheres vão apresentar algum grau da doença ao longo de sua vida. Mulheres com tendência maior, chegando até 33\%. Trata-se de uma doença que tende a evoluir, levando até 11\% das pessoas com varizes podem aos estágios mais avançados (Sociedade Brasileira de Angiologia e de Cirurgia Vascular Regional de São Paulo [SBACV-SP], 2015). Essa tendência pode ser evidenciada no relato de caso de Valente et al (2009), no qual a paciente apresentava um quadro de dermatofibrose devido à estase venosa, agravada pela presença de edemas e quadro flebítico.

De modo similar, o estudo de Leal et al. (2015) demostrou uma prevalência de indivíduos que devido à postura ortostática prolongada, apresentavam fadiga muscular, deterioração dos capilares e edemas. Observa-se que os hábitos de vida, ocupação, sexo do paciente são fatores intrínsecos ao aparecimento de transtornos circulatórios com prejuízo à circulação sanguínea e linfática. Já no estudo de Crisóstomo et al. (2014), embora a amostra do estudo (41 pacientes) apresentasse queixas semelhantes, eles se detiveram em avaliar a melhoria da qualidade de vida relacionada à saúde (QVRS), sintomatologia e estado físico após o tratamento com DLM.

Os 3 estudos abordam intervenções semelhantes, diferenciando-se em suas abordagens e por uma intervenção mecânica (Drenagem Linfática Mecânica) presente no relato de caso. No mais, os 3 enfatizam que o procedimento da DLM ocasionou melhora do quadro clínico de seus pacientes, não havendo diferença significativa nos resultados apesar das diferentes intervenções. O estímulo de linfonodos cervicais inferiores e de linfonodos femorais foi de grande importância para a redução dos edemas e hiperpigmentação, alívio da sintomatologia álgica e fadiga. Portanto, é relevante que haja padronização dessa intervenção (DLM) no tratamento de IVC para que haja menor necessidade de intervenção cirúrgica e farmacológica. 
Ainda abordando patologias venosas, Aldunate et al. (2010) discorre que a diabete, insuficiência venosas ou arterial são contribuem com o aparecimento de lesões ulceradas nos membros inferiores de seus portadores. Algo devidamente explorado no estudo de Azoubel et al (2010), o qual comparou a melhora dos pacientes que apresentavam IVC e hipertensão arterial sistêmica (HAS). A HAS é uma doença que interfere no processo cicatricial ao induzir alteração endotelial, inibição da síntese de colágeno e diminuição do aporte de oxigênio tecidual devido à vasoconstrição.

Em relação aos grupos portadores de IVC e HAS não houve diferenças estatísticas; contudo, o grupo de intervenção, submetido à DLM apresentou diferenças significativas em relação ao grupo controle, submetido apenas aos curativos. $\mathrm{O}$ autor destaca que mais de $70 \%$ de indivíduos com úlcera ativa apresentam prejuízo na função muscular da panturrilha. Portanto, a terapia atua como um reforço muscular que estimula a hemodinâmica, reduzindo o refluxo venoso e estimulando o processo cicatricial.

O linfedema, segundo Godoy et al. (2004) é o excesso de líquido intersticial no espaço tecidual. Ele é um dos sinais da insuficiência do sistema linfático e ocorre quando ele precisa drenar muito além da sua capacidade. O linfedema é classificado em duas categorias: (1) Primário, quando há malformação do sistema linfático, podendo ser subclassificado em congênito, precoce e tardio (dependendo de quando o paciente manifesta os sintomas) e (2) Secundário, decorrentes de causas externas como infecções (filariose), traumas (cirurgias, pancadas) e trombose. (Táboas et al., 2013. Mendes, 2019).

Mendes (2019) ainda comenta que patologias como a insuficiência valvular linfática, hipo/aplasia linfática, estão diretamente ligadas ao surgimento do edema linfático secundário (LS). A hipertensão nos vasos linfáticos e diminuição da contração leva ao acúmulo da linfa, fluido intersticial e proteínas na pele. Com isso, há aumento da pressão osmótica coloidal, gerando o edema. No caso dos LS causados por traumas, como é descrito no estudo de Soligo et al. (2008), a formação dos novos vasos linfáticos é comprometida devido à presença de tecido cicatricial, ocasionando edemas.

Os achados de Kostanoğlu et al. (2019) contribuem ao destacarem que o linfedema é uma condição crônica e progressiva. Sem tratamento adequado, o linfedema pode resultar na proliferação de tecido fibrótico e aumento do risco de feridas e infecções. Em seu estudo, aplica a terapia combinada de descongestionamento (TCD) que inclui a DLM. Esta, resultou em redução significativa do volume das extremidades dos 95 pacientes. Um achado semelhante ao de Soligo et al. (2008) que confirmou a redução do linfedema pela perimetria e volumetria após fazer uso da técnica Godoy\&Godoy na drenagem linfática.

Apesar da melhora dos pacientes após essa terapia, os portadores de linfedema devem manter o cuidado por toda a vida, já que recidivas são frequentes. Manter a TCD, incluindo a drenagem linfática são as medidas mais úteis e eficazes para manter o volume reduzido. Além disso, representam economia por diminuírem a necessidade de analgésicos.

O fibroedema geloide (FEG) consiste numa infiltração edematosa do tecido conjuntivo, seguida de polimerização da substância fundamental amorfa, resultante de uma alteração no meio interno (Brandão et al., 2010). Sendo assim, o fibroedema geloide, mais conhecido como "celulite" pode ser definido como um espessamento não inflamatório das capas subdérmicas. Apesar da existência de vários métodos de tratamento - iontoforese, termoterapia, carboxiterapia, drenagem linfática etc - o estudo de Meyer et al. (2008) destaca que a maioria não apresenta provas de sua efetividade.

Portanto, nesse estudo as 3 pacientes foram submetidas à ressonância magnética (RM) a fim de avaliar a arquitetura subcutânea antes e após o tratamento com drenagem linfática. A DML promoveu a retirada do excesso de líquido presente nos espaços intersticiais, melhorando o processo fibrótico na paciente 1. Além disso, os pacientes 2 e 3 apresentaram menos sinais de estruturas vasculares subdérmicas no exame pós-tratamento apresentou contorno de pele menos ondulado no exame póstratamento, sugerindo diminuição do ingurgitamento venoso.

Segundo Alencar e Ognibeni (2009) as razões que levam a formação de edemas durante o período gestacional não estão plenamente definidas, acredita-se que seja um conjunto de fatores associados à ação hormonal. Hormônios como o estrogênio, cortisol, progesterona e relaxina promovem aumento da permeabilidade capilar, aumento da pressão capilar, compressão de 
válvulas nervosas, diminuição na quantidade de proteínas no sangue e retenção de líquido que ocorre em virtude (Delgado et al., 2017). O edema causa sensações desconfortáveis como dor, fadiga e dormência, por isso há uma preocupação em tratar para tornar a gestação um período mais agradável.

No estudo de Delgado et al. (2017), as 10 gestantes foram submetidas a sessões de DLM. Algumas com mais, outras com menos, mas a redução dos sintomas (edema, dor e fadiga nos membros inferiores) foi unânime; tanto em fase aguda (primeira sessão) quanto na crônica (última sessão). Segundo os autores, os tratamentos de edema gestacional nos membros inferiores têm por finalidade principalmente reduzir os sintomas ao invés de curá-los. Isso se deve principalmente por ser um processo natural durante a gravidez e não ser recomendável a manipulação hormonal durante esse período.

\section{Conclusão}

A drenagem linfática mostrou-se eficiente no tratamento de dermatofibrose, flebite, doença venosa crônica, fibroedema geloide, linfedemas e edemas gestacionais. Uma vez que promove eliminação de líquidos e toxinas e tonificação muscular, os benefícios para a circulação sanguínea são evidentes. Conforme os estudos demonstraram, a DLM promove redução do edema e alívio das dores, sendo de interesse clínico a padronização dessa técnica para que seja aplicada em tratamentos futuros.

Tendo em vista os benefícios supracitados, consideramos proveitoso que os futuros trabalhos que tenham como tema a DLM, abordem o uso dessa intervenção no tratamento de outras patologias, tais como: síndrome pré-menstrual, circulação sanguínea com retorno comprometido, tratamento pós cirurgia plástica, cicatrizes, desconforto pélvico; dentre outras. Ademais, sugerimos que invistam em estudos clínicos a fim de protocolar o uso da drenagem nas mais diversas enfermidades e otimizar o serviço daqueles que aplicam esse tratamento.

\section{Referências}

Aldunate, J. L. C. B., Isaac, C., de Ladeira, P. R. S., Carvalho, V. F., \& Ferreira, M. C. (2010). Úlceras venosas em membros inferiores. Revista de Medicina, 89(3-4), 158-163.

Alencar, R. S., \& Ognibeni, L. C. R. (2009). Uso de drenagem linfática no edema gestacional. Revista Uningá, 21(1).

Andrade, J., Bischoff, L. C., Bonetto, L., Dias, C. P., \& Roncada, C. (2015). Intervenções escolares para redução da obesidade infantil: uma revisão sistemática. Ciência \& Saúde, 8(2), 72-78.

Azoubel, R., Torres, G. D. V., Silva, L. W. S. D., Gomes, F. V., \& Reis, L. A. D. (2010). Efeitos da terapia física descongestiva na cicatrização de úlceras venosas. Revista da Escola de Enfermagem da USP, 44(4), 1085-1092.

Brandão, D. S. M., de Almeida, A. F., Silva, J. C., de Oliveira, R. G. C. Q., de Araújo, R. C., \& Pitangui, A. C. R. (2010). Avaliação da técnica de drenagem linfática manual no tratamento do fibro edema geloide em mulheres. ConScientiae Saúde, 9(4), 618-624.

Cortez, Mejia, D, P, M. Efeitos sistêmicos da drenagem linfática.https://portalbiocursos.com.br/ohs/data/docs/19/50_-_Efeitos_sistYmicos_da _drenagem_linfYtica.pdf.

De-la-Torre-Ugarte-Guanilo, M. C., Takahashi, R. F., \& Bertolozzi, M. R. (2011). Revisão sistemática: noções gerais. Revista da Escola de Enfermagem da USP, 45(5), 1260-1266.

de Godoy, J. R. P., da Silva, V. Z. M., \& de Souza, H. A. (2004). Linfedema: revisão da literatura. Universitas: Ciências da Saúde, 2 (2), $269-282$.

de Franca, C. P., Aguiar, G. F., \& Parra, C. C. (2014). Efeitos Fisiológicos e benefícios da drenagem linfática manual em edema de membros inferiores: Revisão de Literatura. Monografia (Bacharelado em Fisioterapia) Centro Universitário Católico Salesiano Auxilium de Araçatuba, Araçatuba.

França, L. H. G., \& Tavares, V. (2003). Insuficiência venosa crônica. Uma atualização. J Vasc Br, 2(4).

Goroll, Allan H., Mulley, Albert G. (2006) Evaluation of Leg edema. Primary care medicine: office evaluation and management of the adult patient. 5(1)

Guirro, E., \& Guirro, R. R. J. (2002). Fisioterapia Dermato-Funcional: Fundamentos. Recursos e Patologias, 1, 3-23.

Kostanoğlu, A., Ramoğlu, M., \& Güneren, E. (2019). Results of home-based modified combined decongestive therapy in patients with lower extremity lymphedema. Turkish Journal of Medical Sciences, 49(2), 610-616.

Leal, F. D. J., Couto, R. C., Silva, T. P. D., \& Tenório, V. D. O. (2015). Fisioterapia vascular no tratamento da doença venosa crônica. Jornal Vascular Brasileiro, 14(3), 224-230. 
Research, Society and Development, v. 10, n. 4, e14810413968, 2021

(CC BY 4.0) | ISSN 2525-3409 | DOI: http://dx.doi.org/10.33448/rsd-v10i4.13968

Marçal, D. F. D. S., Alexandrino, E. G., Cortez, L. E. R., \& Bennemann, R. M. (2018). Efeitos do exercício físico sobre diabetes mellitus tipo 1: uma revisão sistemática de ensaios clínicos e randomizados. Journal of Physical Education, 29.

Mendes, S. C. G. M. M. (2019). Linfedema: Causas, diagnóstico e tratamento (Doctoral dissertation, Universidade de Coimbra).

Meyer, P. F., Martins, N. M., Martins, F. M., Monteiro, R. A., \& Mendonça, K. M. P. P. D. (2008). Effects of lymphatic drainage on cellulitis assessed by magnetic resonance. Brazilian Archives of Biology and Technology, 51(SPE), 221-224.

Müller, M., Klingberg, K., Wertli, M. M., \& Carreira, H. (2018). Manual lymphatic drainage and quality of life in patients with lymphoedema and mixed oedema: a systematic review of randomised controlled trials. Quality of life research, 27(6), 1403-1414.

Ozolins, B. C., Mendes, A. F., Pinto, L. P., \& Assis, I. B. (2018). Drenagem Linfática Clássica: revisão de literatura. Revista Saúde em Foco, (10), $319-323$.

Pena, J. C. O., \& Macedo, L. B. (2011). Existe associação entre doenças venosas e nível de atividade física em jovens? Fisioterapia em Movimento, 24(1), 147154.

Piccinin, A. M., Mello, P. B., Bem, D. M., Silva, A., \& Rosa, P. V. (2009). Redução do edema em membros inferiores através da drenagem linfática manual: um estudo de caso. Rev Inspirar Mov Saude, 1(2), 10-4.

Pinheiro, P. Causas de inchaços nas pernas e no corpo (retenção de líquidos). https://www.mdsaude.com/nefrologia/ inchacos-edema/.

Rocha, R. S., \& Mejia, D. P. M. A drenagem linfática manual como tratamento do edema no pós-cirúrgico de lipoaspiração: Revisão de literatura.

Rossi, C. E., \& Vasconcelos, F. D. A. G. D. (2010). Peso ao nascer e obesidade em crianças e adolescentes: uma revisão sistemática. Revista Brasileira de Epidemiologia, 13(2), 246-258.

Rg, S. (2012). Drenagem linfática manual como coadjuvante no pós-operatório de abdominoplastia. Revista Presciência, 71-82.

Sociedade Brasileira de Angiologia e de Cirurgia Vascular Regional de São Paulo (SBACV-SP). Insuficiência Venosa Crônica / Varizes dos Membros Inferiores. https://sbacvsp. com.br/insufici encia-venosa-cronica-varizes-dos-membros-inferiores/.

Soligo, C. G., Godoy, J. M. P., Maria de Fátima, G., \& Taglietto, V. R. (2008). Nova técnica de drenagem linfática melhorando o padrão linfocintiligráfico no linfedema traumático: relato de caso. Arq. ciênc. saúde, $42-44$.

Sousa, J. G., et al (2010) Os recursos terapêuticos manuais nos pós-cirúrgicos de cirurgias plásticas abdominais. https://inter fisio.com.br/os-recursosterapeuticos-manuais-nos-poscirurgi cos-de-cirurgias-plasticas-abdominais/.

Táboas, M. I., Torres, A., Popik, I., Casalta, P., Lima, L., \& Caldas, J. (2013). Lymphedema: review and integration of a case report. Revista SPMFR, 21(14), 70-8.

Tacani, Rogério, Tacani, Pascali. Drenagem linfática manual terapêutica ou estética: existe diferença?

Trayes, K. P., Studdiford, J. S., Pickle, S., \& Tully, A. S. (2013). Edema: diagnosis and management. American family physician, 88(2), 102-110.

Valente, F. M., Godoy, M. D. F. G., \& de Godoy, J. M. P. (2009). Drenagem linfática em paciente com dermatofibrose e flebite de membro inferior-relato de caso Lymph drainage in patients with dermatofibrosis and phlebitis of the lower limb-case report. Rev Inst Ciênc Saúde, 27(2), 133-5.

Zanella, B. I., Ruckl, S., \& Voloszin, M. (2011). A importância da drenagem linfática manual no pôs operatório da abdominoplastia. Rev. Lit.[periódico na internet]. 